\title{
Wake-up call for genome scanners
}

According to a recent paper in the American Journal of Human Genetics, most genome-scan studies looking for loci that influence complex human diseases or traits, overestimate how much the loci that are found contribute to such phenotypes. This might not come as news to those quantitative geneticists who map quantitative trait loci (QTL) in domesticated or experimental species, but, for many human geneticists, this finding might come as a rather rude awakening. Importantly, the knock-on effect of such overestimations is that follow-up studies can underestimate the power required to replicate the original study, which might be why so many replication studies come up empty-handed.

The primary goal of a genome scan is to find the genetic loci that influence a particular phenotypic trait. If evidence for a locus is found, it is also very useful to know the locus effect size - how much of the heritability of the phenotype is attributable to variation at that locus. And that's where the problem lies. The position of the locus is estimated by the LOD score, which measures the likelihood of linkage at any given location, but because the LOD score is positively correlated with the effect size of the locus, the location and the heritability of the locus cannot be estimated independently from the same data set.

To study this phenomenon which has been well documented in experimental species - in the context of human genetics, Göring et al. simulated and mathematically analysed a fairly typical genome scanning experiment: the hypothetical population comprises 1,000 families, each with two offspring; the trait being modelled has a heritability of 0.5 , with variable numbers of QTL; genotypic data are available from markers at 2-cM intervals; and there are no complicating factors such as epistatic or gene-environment interactions. But even with this wellbehaved data set, the authors found that there is no way to reliably estimate the heritability effect of any QTL. Their mathematical analysis of the problem shows that the QTL effect is always overestimated, although this bias does fall away with increasing sample size.

So, if a published genome scan contains an estimate of the effect of a particular QTL, calculated from a single data set, it will almost certainly be inflated. When a follow-up study fails to replicate the first report of a QTL, it might be that such a locus exists, but that the follow-up study did not have enough power to detect it - so it's not all bad news. However, the way forward could be daunting. The authors suggest that the most realistic solution is to use independent data sets for the estimation of location and effect size, and that means big samples — very big samples indeed.

Mark Patterson (0) References and links ORIGINAL RESEARCH PAPER Göring, H. H. H. et al. Large upward bias in estimation of locusspecific effects from genomewide scans. Am. J. Hum. Genet. 69, 1357-1369 (2001) FURTHER READING Cardon, L. \& Bell, J. Association study designs for complex diseases. Nature Rev. Genet. 2, 91-99 (2001) WEB SITES

John Blangero's lab: http://www.sfbr.org/sfbr/departments/genetics/ popgen/john_blangero.html

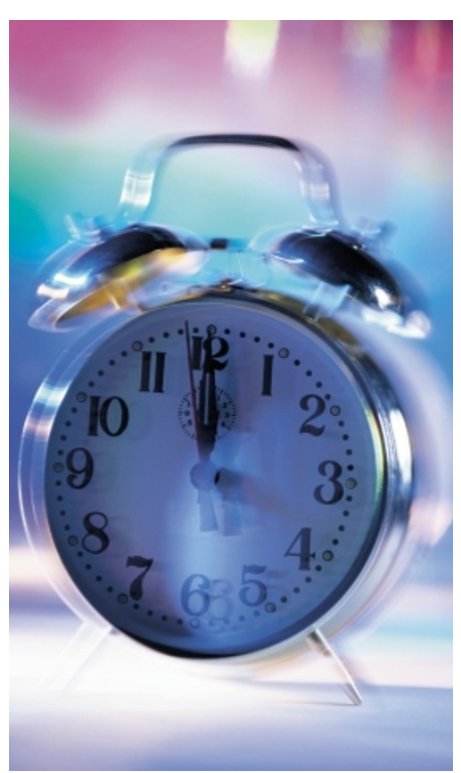

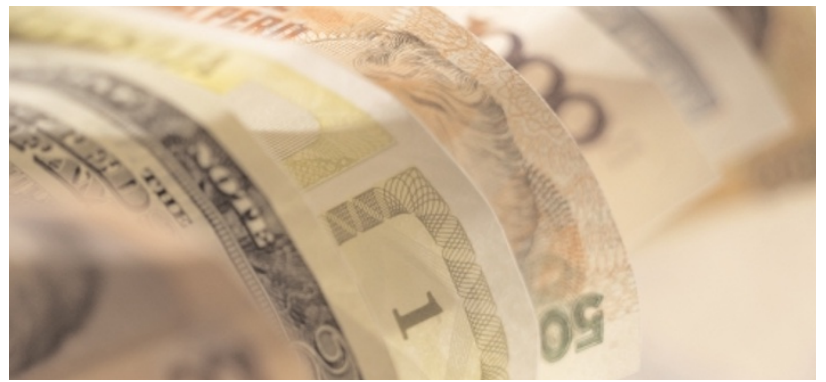

RECOMBINATION

\section{A different exchange-rate mechanism}

Meiosis-specific processes, such as pairing of homologous chromosomes and recombination, are highly conserved, nevertheless there have been conflicting theories about the sequence of events that leads up to recombination. In yeast, it is well established that meiotic exchange can take place in the absence of the synaptonemal complex (SC) - a proteinaceous 'glue' that holds sister chromatids together. However, in a recent issue of Genes and Development, Page and Hawley provide new evidence that, in flies, the SC is essential for the initiation of recombination, and that there might be some unexpected and fundamental differences between meiosis in flies and in yeast.

Previous evidence from Drosophila mutants has shown that the relationship between recombination and the $\mathrm{SC}$ is not the same as it is in yeast. For example, the mei-W68 mutant has a normal SC but does not undergo recombination and the $c(3) G$ mutant eliminates both the $\mathrm{SC}$ and recombination. To investigate whether recombination depends on the SC, Page and Hawley have cloned $c(3) G$ and shown that it encodes an essential component of the SC As expected, C (3)G localizes between the paired homologues, and this localization is altered in mutants that disrupt the SC, confirming its role as an integral part of the SC.

Using C(3)G as a marker, Page and Hawley looked at the SC in mutants in which meiotic exchange is defective. One class of these mutants has reduced frequency of exchange, and the distribution of crossover sites is biased against distal parts of the chromosomes - a phenomenon known as polar effect. Because $\mathrm{C}(3) \mathrm{G}$ is mislocalized in these mutants, the authors conclude that incorrect SC assembly might, at least partially, account for the polar effect. But even in normal cells, crossovers are not randomly distributed along the chromosome because of crossover interference - the suppression of crossovers in the immediate neighbourhood of an established crossover point. In yeast, incorrect assembly of the SC and incomplete pairing of the homologues abolishes interference, but in Drosophila, Page and Hawley show that when the SC is absent because $\mathrm{C}(3) \mathrm{G}$ is mislocalized or non-functional, interference is essentially unaffected.

The picture that emerges is that, in contrast to yeast, which might use the initiation of recombination to align homologous chromosomes, and the SC just to stabilize their pairing, Drosophila needs the SC for the initial alignment of the homologues, without which recombination will not be initiated. It also seems that these two organisms have different ways of controlling interference-in yeast this process is SC dependent, whereas in flies it is SC independent. It remains to be seen which way is more common among other organisms.

Magdalena Skipper

(2) References and links

ORIGinAL RESEARCh PAPER Page, S. L. \& Hawley, R. S. c(3)G encodes a Drosophila synaptonemal complex protein. Genes Dev. 15, 3130-3143 (2001)

ENCYCLOPEDIA OF LIFE SCIENCES Meiosis

WEB SITE

Scott Hawley's lab: http://www.mcb.ucdavis.edu/faculty-labs/hawley/Meiosis.html 